\title{
ATP-induced cardioprotection against myocardial ischemia/reperfusion injury is mediated through the RISK pathway
}

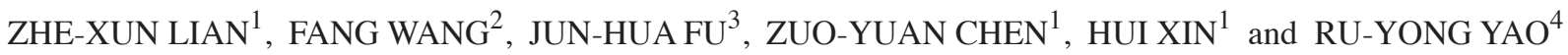 \\ ${ }^{1}$ Department of Cardiology, The Affiliated Hospital of Qingdao University, Qingdao, Shandong 266003; \\ ${ }^{2}$ Department of Cardiology, The Third People's Hospital of Qingdao, Qingdao, Shandong 266000; \\ Departments of ${ }^{3}$ Interventional Medicine and ${ }^{4}$ Central Laboratory, The Affiliated Hospital \\ of Qingdao University, Qingdao, Shandong 266003, P.R. China
}

Received April 14, 2016; Accepted July 25, 2016

DOI: $10.3892 /$ etm.2016.3563

\begin{abstract}
The aim of the present study was to examine the post-infarct acute effect of adenosine-5'-triphosphate (ATP) on myocardial infarction (MI) size as well as its precise molecular mechanism. Sixty New Zealand white male rabbits were exposed to $40 \mathrm{~min}$ of ischemia followed by $180 \mathrm{~min}$ of reperfusion. The rabbits were intravenously administered $3 \mathrm{mg} / \mathrm{kg}$ of ATP (ATP group) or saline (control group) immediately after reperfusion and maintained throughout the first $30 \mathrm{~min}$. The wortmannin+ATP, PD-98059+ATP, and 5-hydroxydecanoic acid (5-HD) sodium salt+ATP groups were separately injected with wortmannin $(0.6 \mathrm{mg} / \mathrm{kg})$, PD-98059 (0.3 mg/kg), and 5-HD (5 mg/kg) $5 \mathrm{~min}$ prior to ATP administration. MI size was calculated as the percentage of the risk area in the left ventricle. Myocardial apoptosis was determined using a TUNEL assay. Western blot analysis was performed to examine the levels of protein kinase B (Akt)/ p-Akt and extracellular signal-regulated kinase (ERK)/p-ERK in the ischemic myocardium, $180 \mathrm{~min}$ after reperfusion. The infarct size was significantly smaller in the ATP group than in the control group $(\mathrm{p}<0.05)$. The infarct size-reducing effect of ATP was completely blocked by wortmannin, PD-98059 and 5-HD. Compared with the control group, cardiomyocyte apoptosis was significantly reduced in the ATP group, while this did not occur in the wortmannin+ATP, PD-98059+ATP and 5-HD+ATP groups. Western blot analysis revealed a higher myocardial expression of p-Akt and p-ERK 180 min following reperfusion in the ATP versus the control group. In conclusion,
\end{abstract}

Correspondence to: Professor Zhe-Xun Lian, Department of Cardiology, The Affiliated Hospital of Qingdao University, 16 Jiangsu Road, Qingdao, Shandong 266003, P.R. China

E-mail: uvhiv75574@163.com

Key words: adenosine-5'-triphosphate, myocardial reperfusion injury, adenosine-5'-triphosphate-dependent potassium channels, reperfusion injury salvage kinase cardioprotection by postischemic ATP administration is mediated through activation of the reperfusion injury salvage kinase (RISK) pathway and opening of the mitochondrial ATP-dependent potassium channels.

\section{Introduction}

Myocardial infarction (MI) is the most common cause of mortality and disability, with a direct correlation between infarct size and prognosis (1). Reperfusion of ischemic tissue is necessary to terminate the processes of ischemic injury that ultimately results in infarction. However, abrupt reperfusion may be associated with severe metabolic and ionic disturbances that can provoke further tissue injury and myocardial cell death [ischemia/reperfusion (I/R) injury] (2). Since reperfusion is the cornerstone of treatment for acute MI, there is great interest in the development of adjunct therapies, which may attenuate reperfusion injury and thereby maximize the benefits of reperfusion $(1,3)$. Adenosine and selective adenosine receptor agonists have been studied extensively for their ability to reduce infarct size and apoptosis (4-8). These effects appear to be mediated via the activation of one or more adenosine receptor subtypes (7). Adenosine-5'-triphosphate (ATP) is rapidly converted to adenosine by ectonucleotidases, and coronary vasodilation caused by exogenous ATP is entirely mediated by adenosine acting on A2A-adenosine receptors (9). We previously demonstrated that cardioprotection of ischemic postconditioning and ATP-postconditioning in rabbits is associated with the activation of adenosine receptors (10).

Previous findings showed that ischemic postconditioning exerts protective effects through the recruitment of prosurvival kinases such as phosphatidylinositol 3-kinase (PI3K)/protein kinase B (PKB)/Akt and the p42/p44 extracellular signalregulated kinase $1 / 2(\mathrm{ERK} 1 / 2)$ pathways [also known as reperfusion injury salvage kinase (RISK) pathway] at the time of reperfusion $(11,12)$. However, whether exogenous ATP can induce postconditioning effects in the myocardium or whether these effects are mediated through a mechanism similar to that of ischemic postconditioning has yet to be fully examined. 
Therefore, in the present study, using an in vivo rabbit model of acute MI, we examined the acute effects of ATP on myocardial infarct size and apoptosis inhibition as well as its precise molecular mechanism involved in the activation of specific survival signals (PI3K/AKT and ERK1/2 pathways).

\section{Materials and methods}

Experimental animals. Sixty male New Zealand white rabbits with a body weight of $2.0-2.5 \mathrm{~kg}$, were used in the present study. The rabbits were housed in a temperature-controlled environment $\left(21 \pm 2^{\circ} \mathrm{C}\right)$ on a 12 -h light/dark cycle (lights on at 06:00). The animals had free access to food and water. Facilities housing the animals were followed guidelines of the AAALAC (the Association for Assessment and Accreditation of Laboratory Animal Care International), accredited at the time of the study. The study protocol was approved by the Ethics Committee of Qingdao University School of Medicine (Qingdao, China).

Reagents. Wortmannin (PI3K inhibitor), PD-98059 (ERK inhibitor), and 5-hydroxydecanoic acid (5-HD) (mitochondrial ATP-dependent potassium ion channel blocker) were purchased from Sigma Chemical Co. (St. Louis, MO, USA). ATP was purchased from the Tianjin Pharmaceutical Group Jiao-Zuo Co. (Tianjin, China). To detect and quantify apoptosis, a terminal deoxynucleotidyl-transferase-mediated dUTP nick end-labeling (TUNEL) assay was performed according to the manufacturer's instructions using a commercially available kit (Roche, Basel, Switzerland). Any other reagents used were of standard analytical grade.

Surgical preparation. Male New Zealand white rabbits were anesthetized with urethane $(5 \mathrm{ml} / \mathrm{kg})$. Surgical procedures were performed aseptically. The left carotid artery was cannulated to monitor arterial pressure, and electrocardiogram (ECG) leads were placed to record the heart rate. A polyethylene catheter (0.9-mm lumen diameter) was inserted into the internal carotid artery and was advanced $1 \mathrm{~cm}$ towards the heart to monitor blood pressure. Blood pressure was measured using a fluid-filled pressure transducer connected to the end of the cannula. Arterial blood pressure and the heart rate were measured via a catheter introduced into the carotid artery. A micromanometer-tipped catheter (SPR-407; Millar Instruments, Houston, TX, USA) was inserted into the left ventricle to record $+\mathrm{dp} / \mathrm{dt}_{\max }$ (representing the cardiac systolic function) as well as - $\mathrm{dp}^{\mathrm{dt} \mathrm{d}_{\max }}$ (representing cardiac diastolic function). Drugs and saline were administered via the ear vein. After left thoracotomy was performed in the third and fourth intercostal spaces in the exposed heart, a $4 / 0$ silk thread was placed beneath the large arterial branch coursing down the middle of the anterolateral surface of the left ventricle. Coronary arterial occlusion and reperfusion were performed by pushing or releasing the snare made from thread. A prominent anterior branch of the left coronary artery was under-run with a 3/0 silk suture, the ends of which were threaded through a 13-mm polypropylene tube to form a snare. After the administration of heparin sodium at a dose of $300 \mathrm{IU} / \mathrm{kg}^{-1}$, regional myocardial ischemia was induced by clamping the snare with hemostat forceps. Reperfusion was instituted by releasing the
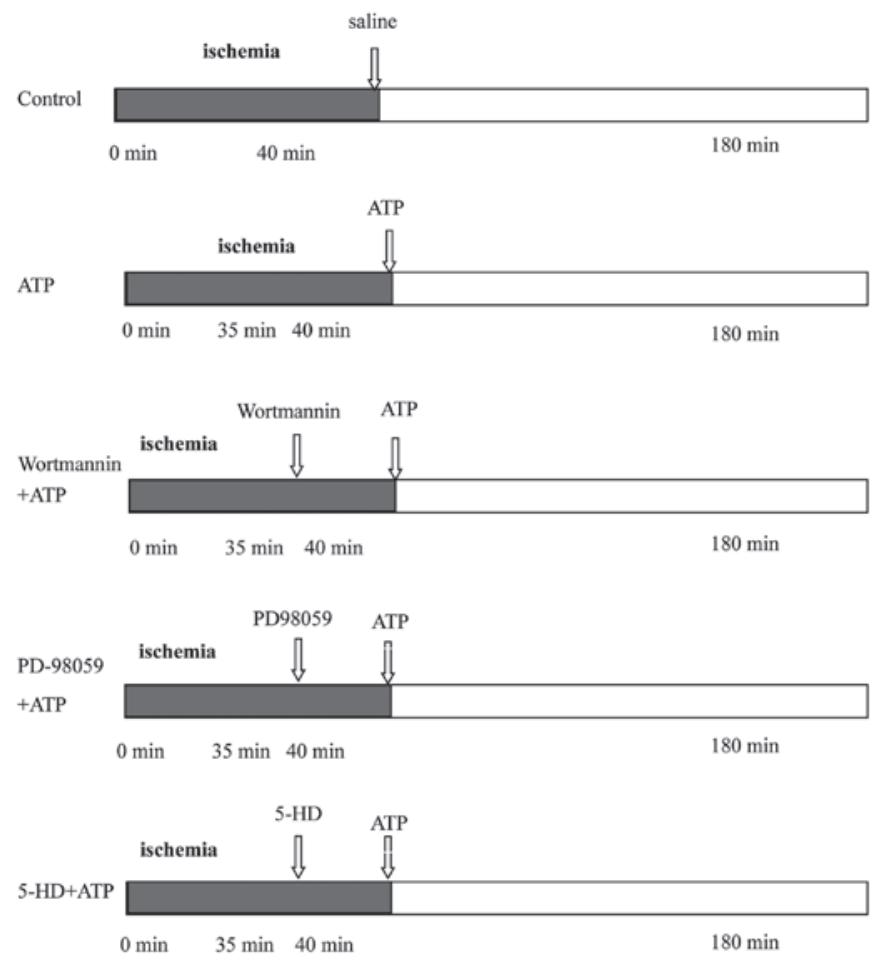

Figure 1. Experimental protocol. All the groups were subjected to a 40-min coronary artery occlusion followed by reperfusion for $180 \mathrm{~min}$. ATP or saline was injected immediately after reperfusion within $30 \mathrm{~min}$. Pharmacological inhibitors (wortmannin, PD98059 and 5-HD) were separately injected 5 min prior to ATP administration. ATP, adenosine-5'-triphosphate; 5-HD, 5-hydroxydecanoic acid.

snare. Coronary arterial occlusion was confirmed by observing cyanosis of the myocardium as well as ST-segment deviation.

Sixty male New Zealand white rabbits underwent $40 \mathrm{~min}$ of coronary occlusion followed by $180 \mathrm{~min}$ of reperfusion, and were then assigned randomly to 5 groups ( $\mathrm{n}=12$ for each group) (Fig. 1). For the control group, $0.9 \% \mathrm{NaCl}$ was administered intravenously immediately after reperfusion and maintained throughout the first $30 \mathrm{~min}$. The ATP group was identical to the control group except that ATP $(3 \mathrm{mg} / \mathrm{kg})$ was administered intravenously and maintained throughout the first $30 \mathrm{~min}$ instead of saline. The wortmannin+ATP, PD-98059+ATP, and 5-HD+ATP groups were identical to the ATP group except that wortmannin (PI3K inhibitor, $0.6 \mathrm{mg} / \mathrm{kg}$ ), PD-98059 (ERK inhibitor, $0.3 \mathrm{mg} / \mathrm{kg}$ ), or 5-HD [mitochondrial ATP-sensitive $\mathrm{K}^{+}\left(\mathrm{mitoK}_{\text {АтР }}\right)$ channel blocker, $5 \mathrm{mg} / \mathrm{kg}$ ] were injected intravenously as a bolus, 5 min prior to initiation of ATP injection in the respective groups.

Analysis of MI size. After the 3-h reperfusion period, the coronary branch was reoccluded and Evans Blue dye solution $(4 \mathrm{ml}, 2 \% \mathrm{w} / \mathrm{v})$ was injected into the left ventricle to distinguish between perfused and non-perfused (myocardium at risk) sections of the heart. The Evans Blue solution stained the perfused myocardium, while the occluded vascular bed was not stained. The rabbits were sacrificed using an intravenous overdose of pentobarbital $(100-200 \mathrm{mg} / \mathrm{kg})$. The heart was excised and sectioned into $4-$ to $5-\mu \mathrm{m}$ thick sections. After removing the right ventricular wall, the area at risk and non-ischemic myocardium were separated by following 
Table I. Hemodynamic parameters.

\begin{tabular}{lcccc}
\hline Group & Heart rate (beats/min) & Mean blood pressure $(\mathrm{mmHg})$ & $+\mathrm{dp} / \mathrm{dt}(\mathrm{mmHg} / \mathrm{sec})$ & $-\mathrm{dp} / \mathrm{dt}(\mathrm{mmHg} / \mathrm{sec})$ \\
\hline Control & $238.8 \pm 7.28$ & $72.5 \pm 4.23$ & $2924.3 \pm 157.69$ & $2325.00 \pm 374.60$ \\
ATP & $240.2 \pm 6.65$ & $71.0 \pm 5.18$ & $4432.17 \pm 221.78^{\mathrm{a}}$ & $4129 \pm 136.90^{\mathrm{b}}$ \\
Wortmannin+ATP & $240.0 \pm 6.13$ & $72.0 \pm 6.9$ & $2872.80 \pm 152.6$ & $2162.00 \pm 270.3$ \\
PD-98059+ATP & $243.2 \pm 7.46$ & $70.5 \pm 6.9$ & $2753.8 \pm 178.25$ & $2074.67 \pm 279.65$ \\
5-HD+ATP & $237.8 \pm 7.08$ & $72.2 \pm 5.98$ & $2783.5 \pm 128.98$ & $2206.33 \pm 197.49$ \\
\hline
\end{tabular}

Data are presented as mean \pm SE. ${ }^{\text {a,b }} \mathrm{P}<0.05$ vs. control, wortmannin, PD-98059 and 5-HD groups. ATP, adenosine-5'-triphosphate; 5-HD, 5-hydroxydecanoic acid.

the line of demarcation between blue-stained and unstained (pink/red) tissue. To distinguish between ischemic and infarcted tissue, the area at risk was cut into small sections and incubated $\left(20 \mathrm{~min}\right.$ at $\left.37^{\circ} \mathrm{C}\right)$ with p-nitro-blue tetrazolium (NBT, $0.5 \mathrm{mg} \mathrm{ml}-1$; Sigma Chemical Co.). In the presence of intact dehydrogenase enzyme systems (normal myocardium), NBT forms a dark blue formazan, while areas of necrosis lack dehydrogenase activity and therefore showed no staining. The area at risk (AAR, area without blue dye) was identified and traced from the enlarged projection (x10) of the photographic slide of each ventricular slice. AAR and IS were determined by computerized planimetry using ImageJ software (Chicago, IL, USA). AAR was expressed as a percentage of the left ventricle and IS was expressed as a percentage of the AAR.

Detection of apoptosis. The detection of apoptotic cells was performed using TUNEL as previously reported (13). The tissue blocks were fixed in $4 \%$ paraformaldehyde and incubated with proteinase K. Fragments of DNA in the tissue sections were analyzed using a TUNEL detection kit (Roche). For each slide, the color images of 10 separate fields were captured randomly and digitized. The cells with clear nuclear labeling were defined as TUNEL-positive cells. The apoptotic index (AI) was calculated as the number of TUNEL-positive cells/total number of myocytes x 100 .

Western blot analysis. Western blot analysis was performed to assess the levels of Akt and p-Akt, as well as ERK and p-ERK in the ischemic area of the myocardium following 180-min reperfusion. Hearts were excised, and transmural samples weighing 100-200 mg were obtained from the center of the left ventricular (LV) ischemic region. The border of the ischemic region was defined by the distribution of cyanosis and was marked on the epicardium in ink. Tissue samples obtained after perfusion were quickly frozen in liquid nitrogen and stored at $-80^{\circ} \mathrm{C}$ until the assays were performed. The samples were weighed, homogenized, and used for different measurements. Proteins were separated and transferred to membranes using standard protocols, after which the phosphorylation (activation) of Akt and ERK and total levels of Akt and ERK were assessed using antibodies against each protein, and then analyzed by SDS-PAGE immunoelectrophoresis.

Statistical analysis. Data were expressed as means $\pm \mathrm{SE}$ and analyzed using SPSS 17.0 software (Chicago, IL, USA).
Table II. Comparison of infarct size and AI at the end of reperfusion.

\begin{tabular}{lcc}
\hline Group & Infarct size $(\%)$ & AI $(\%)$ \\
\hline Control & $29.10 \pm 2.94$ & $27.00 \pm 5.76$ \\
ATP & $12.79 \pm 1.87^{\mathrm{a}}$ & $10.33 \pm 5.96^{\mathrm{b}}$ \\
Wortmannin+ATP & $26.54 \pm 2.71$ & $20.67 \pm 4.32$ \\
PD-98059+ATP & $27.93 \pm 3.18$ & $25.50 \pm 4.85$ \\
5-HD+ATP & $26.04 \pm 4.03$ & $21.17 \pm 3.60$ \\
\hline
\end{tabular}

Data are presented as mean $\pm \mathrm{SE}$. a,b $\mathrm{P}<0.05$ vs. control, wortmannin, PD-98059 and 5-HD groups. AI, apoptotic index; ATP, adenosine5'-triphosphate; 5-HD, 5-hydroxydecanoic acid.

Independent samples t-test and one-way ANOVA were used to compare data with post-hoc analysis using Bonferroni's post-hoc test. Differences at $\mathrm{p}<0.05$ were considered statistically significant.

\section{Results}

Physiological findings. Table I shows the hemodynamic parameters that may influence the infarct size. There were no significant differences in blood pressure or heart rate in the five groups at $180 \mathrm{~min}$ after reperfusion. However, $180 \mathrm{~min}$ after reperfusion, $\pm \mathrm{dp} / \mathrm{dt}$ was significantly improved in the ATP group as compared to the remaining four groups.

Infarct size. As shown in Table II, the percentage of infarct size in the area at risk was significantly reduced in the ATP group $(12.79 \pm 1.87 \%, \mathrm{n}=6)$, as compared with the saline control group $(29.10 \pm 2.94 \%, n=6)$. However, pretreatment with wortmannin $(n=6), P D-98059(n=6)$, or 5-HD $(n=6)$ completely eliminated the infarct size-reducing effect of ATP $(26.54 \pm 2.71,27.93 \pm 3.18$ and $26.04 \pm 4.03 \%$, respectively).

Myocardial apoptosis after reperfusion. There was increased apoptosis $180 \mathrm{~min}$ after reperfusion in all the groups, except for the ATP group (Table II), indicating that ATP significantly reduced myocardial apoptosis during early reperfusion. Treatment with wortmannin, PD98059, or 5-HD significantly attenuated the anti-apoptotic effects of ATP. 
A

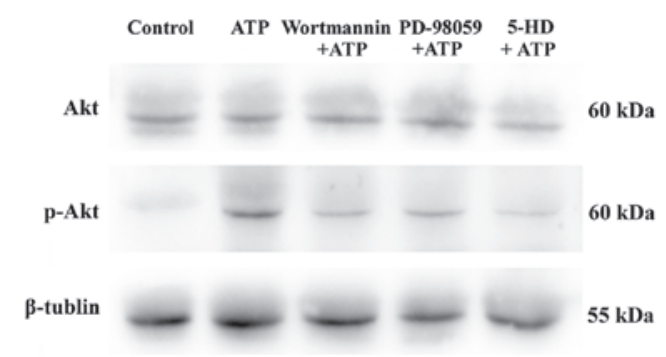

Akt ( arbitrary units)
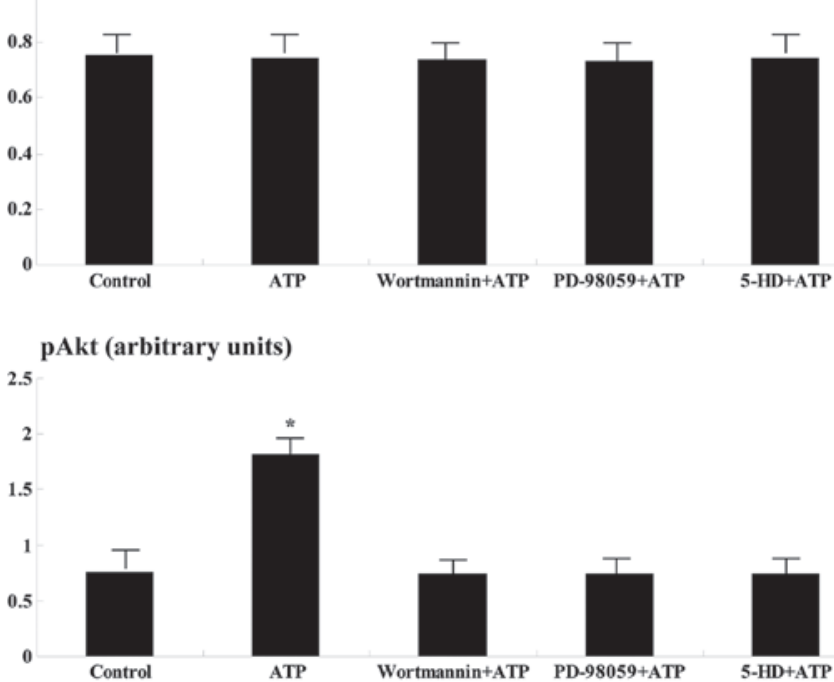

B

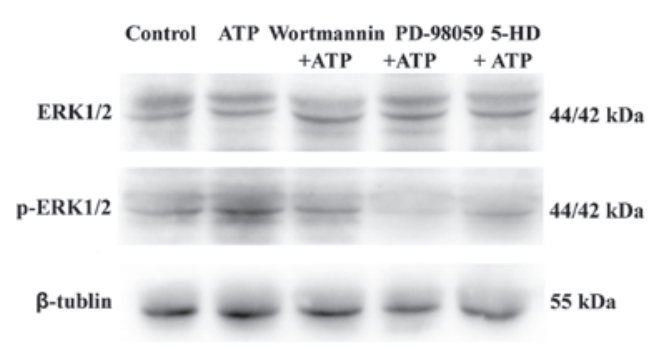

ERK1/2 (arbitrary units)
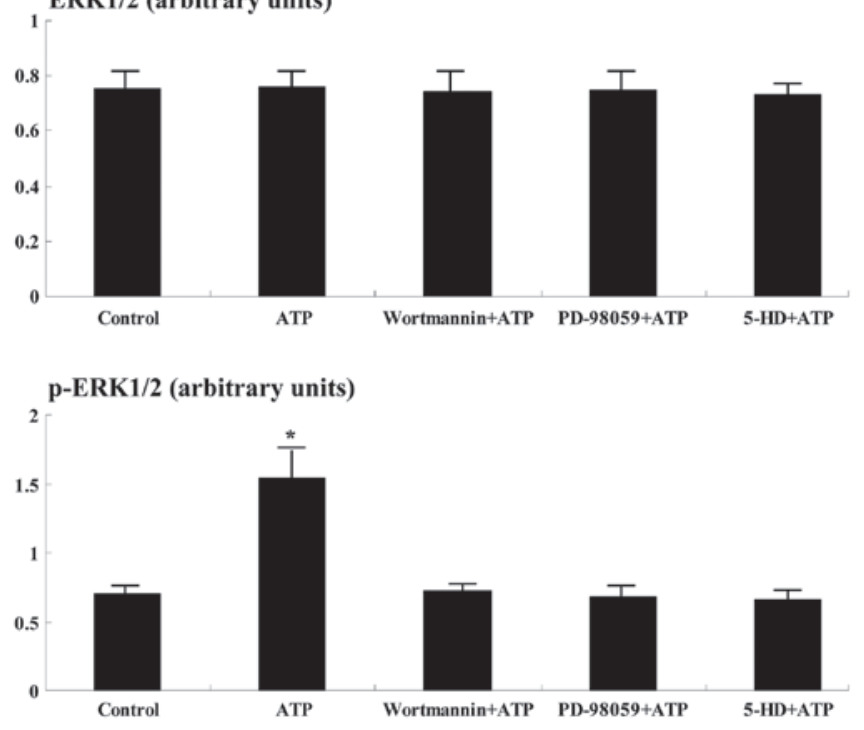

Figure 2. Western blot analysis of (A) myocardial Akt and p-Akt expression in the five groups at 180 min after reperfusion, and (B) myocardial ERK1/2 and p-ERK1/2 expression in the five groups at $180 \mathrm{~min}$ after reperfusion. Values are expressed as mean $\pm \mathrm{SE}$; $\mathrm{n}=6$. Data were normalized to $\beta$-tubulin, as the internal control. ERK, extracellular signal-regulated kinase. ${ }^{*} \mathrm{P}<0.05$ vs. the remaining four groups.

Western blot analysis. One hundred eighty minutes after reperfusion, the levels of p-Akt and p-ERK were significantly upregulated in the ischemic area of the ATP group when compared to the remaining four groups ( $<<0.05$; Fig. 2$)$.

\section{Discussion}

ATP and adenosine are potent coronary vasodilators. Similarly, exogenous ATP is almost completely metabolized to adenosine during a single passage through the heart (14). In humans, the intracoronary administration of ATP causes vasodilation, which is accompanied by an increase in the concentration of coronary sinus adenosine (15). Thus, ATP, as well as adenosine, may be used as cardioprotective agents.

MI is typically associated with apoptosis and the progressive loss of cardiomyocytes caused by apoptosis plays a critical role in cardiac dysfunction after acute MI (16). The present study clearly demonstrates that the intravenous administration of ATP may significantly attenuate cardiomyocyte apoptosis and reduce infarct size. The underlying mechanism may be associated with the inhibition of oxidation stress, upregulation of Bcl-2-encoding mRNA and downregulation of caspase-3 mRNA. Using a swine I/R model Vilahur et al have demonstrated that ischemic postconditioning prevents execution of apoptosis (via Bcl-2 and caspase-3), supporting our observation of the effect induced by ATP administration (17).

Activation of the PI3K/Akt and MEK1/2-ERK1/2 pathways is involved in the infarct size-reducing effect during ischemic postconditioning, as prosurvival signals (18-20). Our observations suggest that ATP treatment during reperfusion activates the PI3K/Akt and MEK1/2-ERK1/2 pathways in the ischemic area. Therefore, since ATP treatment during reperfusion attenuates cardiomyocyte apoptosis and reduces infarct size through activation of the prosurvival signaling pathways such as PI3K/Akt and MEK1/2-ERK1/2, the infarct size-reducing effects of ATP were mediated through a mechanism similar to that of ischemic postconditioning. It has been reported that the adenosine A1/A2 agonist, 5'-N-ethylcarboxamidoadenosine (NECA) and bradykinin can limit infarction when administered at reperfusion in rabbits through a common signaling pathway that includes PI3K, nitric oxide (NO), and ERK (21). The infarct size-reducing effect of ATP is suggested to be due to the activation of PI3K and Akt and subsequent phosphorylation of endothelial nitric oxide synthase (eNOS). Cohen et al previously found that the protective effect of protein kinase $\mathrm{G}$ (PKG) activator during reperfusion can be blocked by $\mathrm{A} 2 \mathrm{~b}$ adenosine receptor, ERK, or PI3K blockers (22).

Concerning the mitoK $_{\text {ATP }}$ channels, it has been reported that either ischemic preconditioning or postconditioning is an effective cardioprotective intervention in rabbits, involving a 
protective mechanism of NO production as well as mitoK ${ }_{\text {ATP }}$ channel opening $(23,24)$. In the present study, the infarct sizereducing effect of ATP was eliminated by pretreatment with 5-HD, a mitoK ${ }_{\text {АTP }}$ channel blocker, suggesting that the infarctreducing effect by ATP was mediated by opening the mitoK $\mathrm{ATP}_{\mathrm{ATP}}$ channels. Since NO has been reported to open mitoK ${ }_{\text {ATP }}$ channels (25), it is possible that ATP activates the PI3K/Akt and eNOS pathways, opens mitoK $\mathrm{ATP}_{\text {TP }}$ channels, and reduces myocardial infarct size. Furthermore, administration of ATP may increase $\mathrm{NO}$ production in the heart (26) and reduce the extent of no-reflow and infarct size (27). As NO and adenosine are important in cardioprotection, the administration of ATP may induce postconditioning in myocardium.

In conclusion, our findings demonstrate that ATP administration immediately after reperfusion reduces myocardial infarct size and exerts a significant cardioprotective effect against I/R injury by inhibiting apoptosis and improving LV function. Cardioprotection by postischemic ATP administration is mediated through activation of the reperfusion injury salvage kinase pathway and opening of the mitoK $\mathrm{K}_{\text {ATP }}$ channels.

Clinical implications. Pharmacological postconditioning is a more practical strategy than preconditioning for treating MI, because it is difficult to predict the timing precisely when acute MI occurs. Previously, however, no pharmacologic adjunctive therapy in patients with acute MI undergoing reperfusion therapy was clearly demonstrated to reduce infarct size and improve clinical outcomes except for adenosine (28-30). Furthermore, the intravenous infusion of ATP may have fewer side effects and be safer than adenosine (31). Therefore, in the clinical setting, pharmacological postconditioning may be induced with exogenous ATP as shown in the present study and may be an alternative strategy for the treatment of acute MI during reperfusion, although further clinical investigations are necessary.

Limitations and future investigations. The major limitation of the present study was that our results did not reveal the protective effect of ATP on preventing myocardial apoptosis during prolonged reperfusion and its optimal timing and dosing. Furthermore, different mechanisms of action for adenosine and ATP may exist. A parallel experimental approach based on the administration of adenosine and adenosine receptor inhibitors may have been of interest to reveal potential differences in the cardioprotective mechanisms.

In conclusion, cardioprotection by postischemic ATP administration is mediated through activation of the reperfusion injury salvage kinase (RISK) pathway and opening of the mitochondrial ATP-dependent potassium channels. However, the results of the present study remain to be verified in future investigations.

\section{Acknowledgements}

We would like to thank Mrs. Nini Gao for her expert editorial assistance and help with manuscript preparation. The present study was supported by the Youth Research Development Funds of the Affiliated Hospital of Qingdao University (200804).

\section{References}

1. Yellon DM and Baxter GF: Protecting the ischaemic and reperfused myocardium in acute myocardial infarction: distant dream or near reality? Heart 83: 381-387, 2000.

2. Hearse DJ and Bolli R: Reperfusion induced injury: manifestations, mechanisms, and clinical relevance. Cardiovasc Res 26: 101-108, 1992.

3. Yellon DM and Baxter GF: Reperfusion injury revisited: is there a role for growth factor signaling in limiting lethal reperfusion injury? Trends Cardiovasc Med 9: 245-249, 1999.

4. Kuno A, Solenkova NV, Solodushko V, Dost T, Liu Y, Yang XM, Cohen MV and Downey JM: Infarct limitation by a protein kinase $\mathrm{G}$ activator at reperfusion in rabbit hearts is dependent on sensitizing the heart to $\mathrm{A} 2 \mathrm{~b}$ agonists by protein kinase $\mathrm{C}$. Am J Physiol Heart Circ Physiol 295: H1288-H1295, 2008.

5. Urmaliya VB, Pouton CW, Ledent C, Short JL and White PJ: Cooperative cardioprotection through adenosine A1 and A2A receptor agonism in ischemia-reperfused isolated mouse heart. J Cardiovasc Pharmacol 56: 379-388, 2010.

6. Kin H, Zatta AJ, Lofye MT, Amerson BS, Halkos ME, Kerendi F, Zhao ZQ, Guyton RA, Headrick JP and Vinten-Johansen J: Postconditioning reduces infarct size via adenosine receptor activation by endogenous adenosine. Cardiovasc Res 67: 124-133, 2005.

7. Regan SE, Broad M, Byford AM, Lankford AR, Cerniway RJ, Mayo MW and Matherne GP: A1 adenosine receptor overexpression attenuates ischemia-reperfusion-induced apoptosis and caspase 3 activity. Am J Physiol Heart Circ Physiol 284: H859-H866, 2003

8. Philipp S, Yang XM, Cui L, Davis AM, Downey JM and Cohen MV: Postconditioning protects rabbit hearts through a protein kinase $\mathrm{C}$-adenosine A2b receptor cascade. Cardiovasc Res 70: 308-314, 2006.

9. Erga KS, Seubert CN, Liang HX, Wu L, Shryock JC and Belardinelli L: Role of $\mathrm{A}(2 \mathrm{~A})$-adenosine receptor activation for ATP-mediated coronary vasodilation in guinea-pig isolated heart. Br J Pharmacol 130: 1065-1075, 2000.

10. Lian ZX, Liu F, Liu S, Xin H, Chen ZY, Tian JH, An Y and Cai SL: Cardioprotection of ischemic postconditioning and ATP-postconditioning in rabbits is associated with the activation of adenosine receptors. Eur Heart J 27 (Suppl 1): 72, 2006.

11. Hausenloy DJ, Tsang A and Yellon DM: The reperfusion injury salvage kinase pathway: a common target for both ischemic preconditioning and postconditioning. Trends Cardiovase Med 15: 69-75, 2005.

12. Bopassa J-C, Ferrera R, Gateau-Roesch O, Couture-Lepetit E and Ovize M: PI 3-kinase regulates the mitochondrial transition pore in controlled reperfusion and postconditioning. Cardiovasc Res 69: 178-185, 2006.

13. Kin H, Wang NP, Mykytenko J, Reeves J, Deneve J, Jiang R, Zatta AJ, Guyton RA, Vinten-Johansen J and Zhao ZQ: Inhibition of myocardial apoptosis by postconditioning is associated with attenuation of oxidative stress-mediated nuclear factor-kappa B translocation and TNF alpha release. Shock 29: 761-768, 2008.

14. Fleetwood G, Coade SB, Gordon JL and Pearson JD: Kinetics of adenine nucleotide catabolism in coronary circulation of rats. Am J Physiol 256: H1565-H1572, 1989.

15. Nanto S, Kitakaze M, Takano Y, Hori M and Nagata S: Intracoronary administration of adenosine triphosphate increases myocardial adenosine levels and coronary blood flow in man. Jpn Circ J 61: 836-842, 1997.

16. Olivetti G, Quaini F, Sala R, Lagrasta C, Corradi D, Bonacina E, Gambert SR, Cigola E and Anversa P: Acute myocardial infarction in humans is associated with activation of programmed myocyte cell death in the surviving portion of the heart. J Mol Cell Cardiol 28: 2005-2016, 1996.

17. Vilahur G, Cubedo J, Casani L, Padro T, Sabate-Tenas M, Badimon JJ and Badimon L: Reperfusion-triggered stress protein response in the myocardium is blocked by post-conditioning. Systems biology pathway analysis highlights the key role of the canonical aryl-hydrocarbon receptor pathway. Eur Heart J 34: 2082-2093, 2013.

18. Heusch G, Boengler K and Schulz R: Cardioprotection: nitric oxide, protein kinases, and mitochondria. Circulation 118: 1915-1919, 2008

19. Goodman MD, Koch SE, Fuller-Bicer GA and Butler KL: Regulating RISK: a role for JAK-STAT signaling in postconditioning? Am J Physiol Heart Circ Physiol 295: H1649-H1656, 2008. 
20. Zhu M, Feng J, Lucchinetti E, Fischer G, Xu L, Pedrazzini T, Schaub MC and Zaugg M: Ischemic postconditioning protects remodeled myocardium via the PI3K-PKB/Akt reperfusion injury salvage kinase pathway. Cardiovasc Res 72: 152-162, 2006.

21. Yang XM, Krieg T, Cui L, Downey JM and Cohen MV: NECA and bradykinin at reperfusion reduce infarction in rabbit hearts by signaling through PI3K, ERK, and NO. J Mol Cell Cardiol 36: 411-421, 2004

22. Cohen MV, Yang XM, Liu Y, Solenkova NV and Downey JM: Cardioprotective PKG-independent NO signaling at reperfusion. Am J Physiol Heart Circ Physiol 299: H2028-H2036, 2010.

23. Hide EJ and Thiemermann C: Limitation of myocardial infarct size in the rabbit by ischaemic preconditioning is abolished by sodium 5-hydroxydecanoate. Cardiovasc Res 31: 941-946, 1996.

24. Yang XM, Proctor JB, Cui L, Krieg T, Downey JM and Cohen MV: Multiple, brief coronary occlusions during early reperfusion protect rabbit hearts by targeting cell signaling pathways. J Am Coll Cardiol 44: 1103-1110, 2004

25. Han J, Kim N, Joo H, Kim E and Earm YE: ATP-sensitive K(+) channel activation by nitric oxide and protein kinase $\mathrm{G}$ in rabbit ventricular myocytes. Am J Physiol Heart Circ Physiol 283: H1545-H1554, 2002.

26. Burnstock G: Overview: purinergic receptors. In: Role of adenosine and adenosine nucleotides in the biological system. Imai S and Nakazawa M (eds). Elsevier Science, Amsterdam, pp1-6, 1991.
27. Komamura K, Ito H, Takiuchi S, Iwakura K, Maruyama A, Masuyama T, Minamino T, Node K, Kitakaze M and Hori M: Transient intracoronary infusion of ATP after reperfusion reduces the extent of no-reflow and infarct size in dogs. J Am Coll Cardiol 25: 227A-228A, 1995.

28. Mahaffey KW, Puma JA, Barbagelata NA, DiCarli MF, Leesar MA, Browne KF, Eisenberg PR, Bolli R, Casas AC, Molina-Viamonte $\mathrm{V}$, et al: Adenosine as an adjunct to thrombolytic therapy for acute myocardial infarction: results of a multicenter, randomized, placebo-controlled trial: the Acute Myocardial Infarction STudy of ADenosine (AMISTAD) trial. J Am Coll Cardiol 34: 1711-1720, 1999.

29. Ross AM, Gibbons RJ, Stone GW, Kloner RA and Alexander RW; AMISTAD-II Investigators: A randomized, double-blinded, placebo-controlled multicenter trial of adenosine as an adjunct to reperfusion in the treatment of acute myocardial infarction (AMISTAD-II). J Am Coll Cardiol 45: 1775-1780, 2005.

30. Kloner RA, Forman MB, Gibbons RJ, Ross AM, Alexander RW and Stone GW: Impact of time to therapy and reperfusion modality on the efficacy of adenosine in acute myocardial infarction: the AMISTAD-2 trial. Eur Heart J 27: 2400-2405, 2006.

31. Miyagawa M, Kumano S, Sekiya M, Watanabe K, Akutzu H, Imachi T, Tanada S and Hamamoto K: Thallium-201 myocardial tomography with intravenous infusion of adenosine triphosphate in diagnosis of coronary artery disease. J Am Coll Cardiol 26: 1196-1201, 1995. 